\title{
Multiscale Structural Architectures of Novel Sulfur Copolymer Composite Cathodes for High-Energy Density Li-S Batteries Studied by Analytical Multimode STEM Imaging and Tomography
}

\author{
V.P. Oleshko ${ }^{1,2}$, A. Herzing ${ }^{1}$, J. Kim ${ }^{1}$, J. Schaefer ${ }^{1}$, C. Soles ${ }^{1}$, J.J. Griebel ${ }^{3}$, W.J. Chung ${ }^{3}$, A.G. Simmonds ${ }^{3}$, \\ J. Pyun ${ }^{3}$ \\ ${ }^{1}$ Material Measurement Laboratory, National Institute of Standards and Technology, Gaithersburg, MD \\ 20899 \\ ${ }^{2}$ Department of Materials Science and Engineering, University of Maryland, College Park, MD 20742 \\ ${ }^{3}$ Department of Chemistry \& Biochemistry, University of Arizona, Tucson, AZ 85721
}

Li-S rechargeable batteries are considered to be a promising light-weight, low-cost, and environmentally friendly candidate for next generation energy storage owing to high theoretical specific capacity of 1,672 $\mathrm{mAh} / \mathrm{g}$ and high specific energy of $2,567 \mathrm{Wh} / \mathrm{kg}$, which is 5 times that of current Li-ion technology. However, practical use of Li-S batteries remains limited because they suffer from gradual capacity fading caused by insulating properties of sulfur and polysulfide shuttle. Recently, poly(sulfur-random-(1,3diisopropenylbenzene) (poly(S-r-DIB)) copolymers have been introduced for their use as active materials in cathodes for Li-S batteries, and were found to be capable of realizing enhanced capacity retention (1005 $\mathrm{mAh} / \mathrm{g}$ at 100 cycles) and a five-fold increase in lifetime (over 500 cycles) as compared to conventional sulfur-carbon cathodes [1,2]. These materials are typically organized in a rough hierarchical 3D architecture which contains multiple components and is quite challenging to understand and characterize.

Herein, we employ multimode analytical STEM imaging coupled with multivariate statistical analysis (MSA) and electron tomography (ET) to investigate the morphology and compositions of the composite cathodes created by combining the poly(S-r-DIB) copolymers with varying DIB content ( $0-50 \%$ by mass) and Timcal Super C65 conductive carbons as well as their structural transformations under cycling in relation to the electrochemical performance, impedance, electrical conductivity, and physico-mechanical stability. Multimode STEM imaging was carried out by simultaneously acquiring HAADF, BF, low-angle and medium-angle ADF signals (FIG. 1a-c) which contain a wealth of information regarding the scattering characteristics of the various phases present and display strong contrast variations [3]. We subsequently analyzed these data with MSA in order to isolate statistically significant differences between spatial regions and produce an unbiased phase classification (FIG.1d). STEM ET data was also collected using small convergence angles of the incident beam down to $4 \mathrm{mrad}$ in order to keep the entire sample in focus even in very thick specimen areas up to several $\mu \mathrm{m}$ (FIG. 1e). Ideally, by recording multiple imaging and spectroscopic signals and analyzing correlations between the signal intensity and structure and elemental compositions, one can noninvasively identify and ultimately quantify all phases (components) in such multiscale structures with complex multifractal morphology. High-tilt-angle STEM ET indicates that aggregated $20 \mathrm{~nm}$ to $60 \mathrm{~nm}$ onion-like Super C65 carbon particles form conductive 3D percolation networks over the cathode as well as extended mesoscale and nanoscale pore structures (FIG. 1f, g). Driftcorrected XEDS/EELS STEM spectral imaging (SI) (FIG. 2) allowed us to evaluate uniformity of compositional distributions within the copolymers and local elemental variations at electroactive poly(S$r$-DIB) - carbon interfaces. In addition, as a result of the improved physico-mechanical stability of the composite cathodes as compared to the conventional sulfur-carbon cathode, structural analyses of the poly(S-r-DIB) - carbon interfaces indicate usually intimate contacts around the carbon nanoparticles which also display a short-range ordering with graphitic-like outer shells and mixed $\mathrm{sp}^{2} / \mathrm{sp}^{3}$-bonding. 
References: [1] W.J. Chung, et al, Nature Chemistry 5 (2013) 518. [2] A.G. Simmonds, et al, J. ACS Macro Lett. 3 (2014), 229. [3] V.P. Oleshko, et al, Nanoscale 6 (2014) 11756.

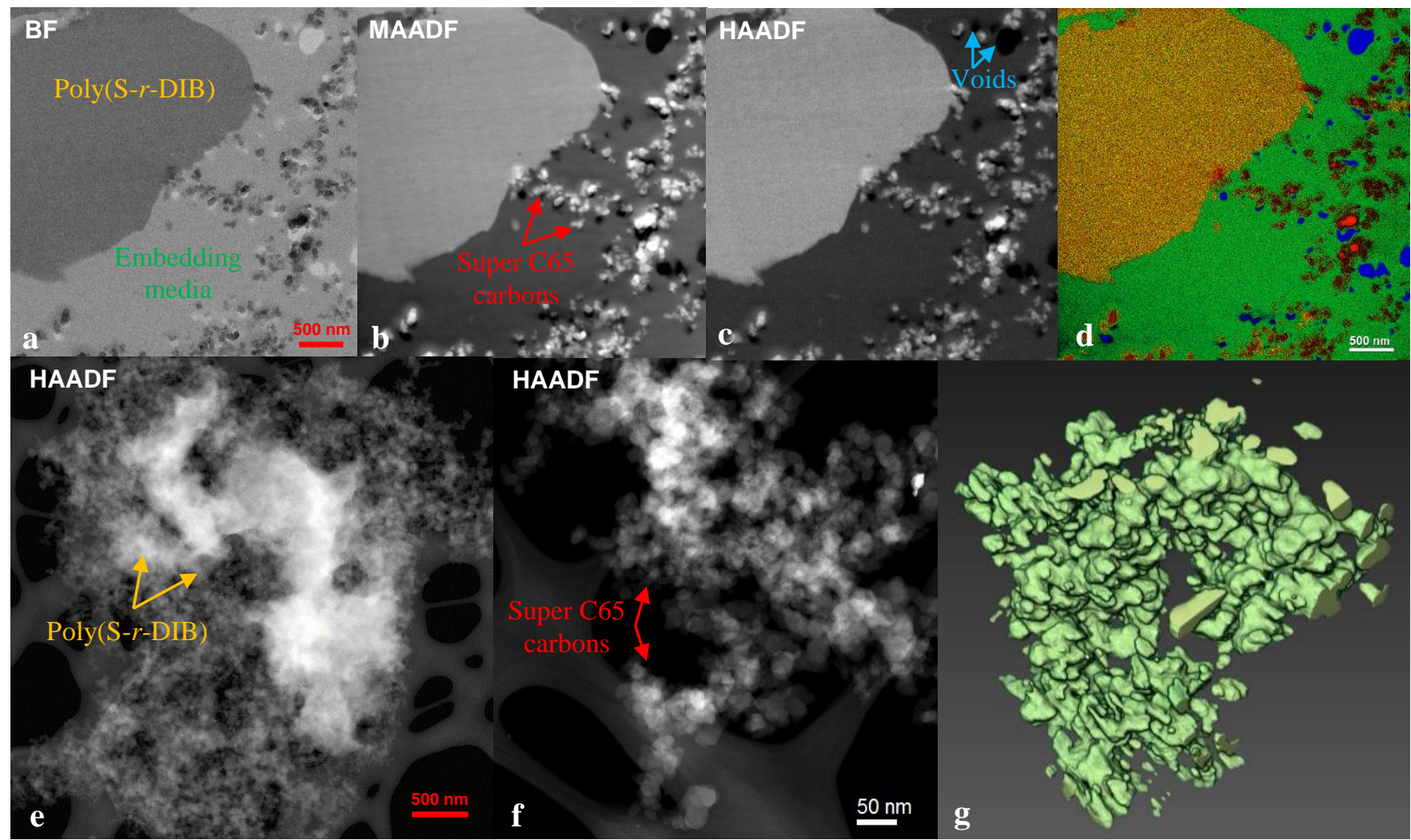

Figure 1. (a-d) Multimode STEM of a thin section of a poly(S-r-DIB 10 -w $\%$ ) copolymer - carbon composite cathode: (a) BF, (b) medium-angle ADF, (d) HAADF, (d) Composite phase image obtained by MSA of a-c. (e) $\mathrm{C}_{\text {s }}$ probe-corrected HAADF STEM and (f, g) high-tilt-angle ET of the cathode powder, a single frame extracted from a tilt series; (g) the reconstructed and segmented 3D view of the cathode structure showing random percolation networks of conductive carbons and extended pores.

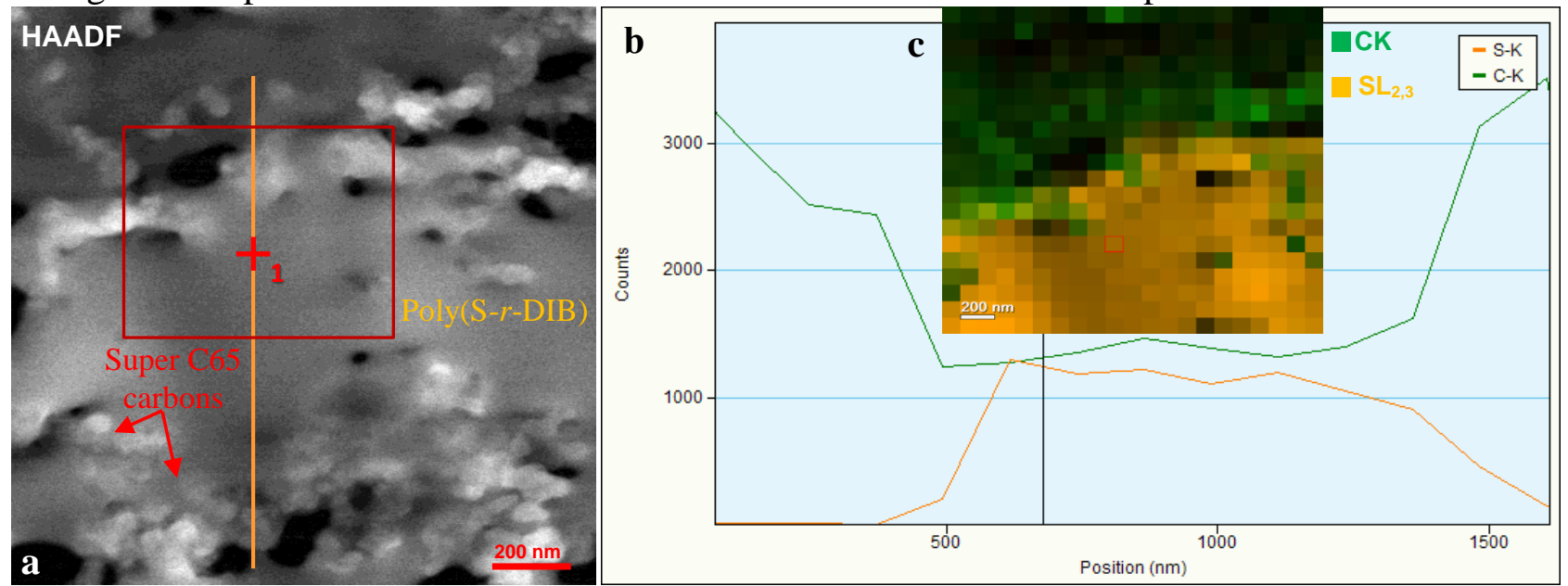

Figure 2. (a) HAADF STEM and (b, c) EDX/EEL STEM SI of a thin section of the $S-r$-DIB $10 \%$ copolymer-carbon composite cathode. (b) X-ray spectral line profile along orange line in (a). The point 1 marked by red cross in (a) corresponds to black line in (b). (c) overlaid C K- and $\mathrm{S} \mathrm{L} \mathrm{L}_{2,3}$ EELS maps acquired in the area marked by red box in (a). 